13

\title{
A novel method of plasmon coupled optical waveguide for aerosol sensor*
}

\author{
(c) Sushil Kumar ${ }^{1}$, Gaurav Sharma ${ }^{2}$, Gulab Chand Yadav², Vivek Singh ${ }^{2}$, and Abhay Kumar Singh ${ }^{2}$ \\ ${ }^{1}$ Department of Physics, Sri Shankar College Sasaram (Constituent Body of Veer Kunwar Singh University Ara), \\ Rohtas, Bihar 821115, India \\ ${ }^{2}$ Department of Physics, Institute of Science, Banaras Hindu University, \\ Varanasi, Uttar Pradesh 221005 India \\ e-mail: skumarmaurya85@gmail.com
}

Received January 09, 2021

Revised July 07, 2021

Accepted July 28, 2021

The tropospheric aerosol characterization is important for understanding environmental process as well as human health safety. In this study, the surface plasmon resonance sensor is proposed for the detection of ammonium sulfate which is an important component of aerosols. The reflectivity of proposed sensor waveguides is calculated as a function of the incident angle using the transfer matrix method. The performance of proposed sensors having sol-gel film of tetraethylorthosilane and thymol blue are studied and compared. Firstly, the dispersion characteristics are obtained for different modes, and it is observed that the modes are tightly bound in thymol blue waveguide in comparison to tetraethylorthosilane waveguide. Further, the magnetic field distributions are also studied and compared for proposed waveguide sensor configurations. It is found that the sol-gel film of thymol blue waveguide provides high sensitivity while tetraethylorthosilane waveguide shows high figure of merit.

Keywords: aerosol, surface plasmon resonance sensor; reflectivity, sensitivity, detection accuracy.

* Полный текст статьи опубликован в „Optics and Spectroscopy“ 2021 V. 129. N 11. 